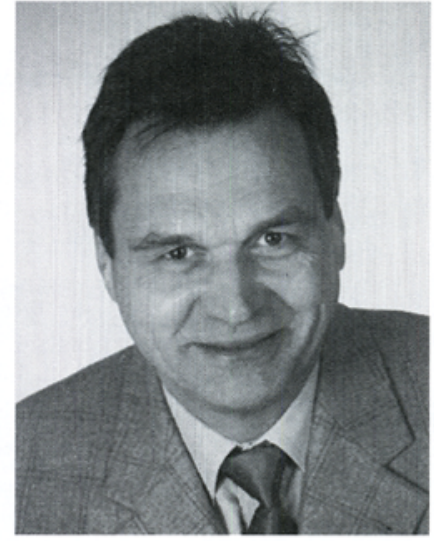

Uwe Fichtner

\section{Virtuelle Kartensammlungen}

Von jedem Ort aus und zu jeder Zeit rasch und ohne die bürokratischen Hemmnisse nationaler Schranken auf das Wissen der Welt und das Kulturgut historischer Kartenschätze zurückgreifen zu können - dieser Vision ist das EU Projekt "Digitale Historische Karten" ein gutes Stück näher gekommen. Reinhard Zölitz-Möller, Jörg Hartleib, Bernd Röber und Hannelore Sattler berichten in diesem Heft, wie eine große Anzahl von teilweise über 300 Jahre alte historische Karten in einer Datenbank archiviert worden sind, die nun über das Internet für die Óffentlichkeit zugänglich ist. Jederzeit ist diese virtuelle Kartensammlung zu betreten Iwww.dhm.Im.sel, ohne sich an leidige O'ffnungszeiten halten zu müssen, und jedermann kann in ihrem Fundus stöbern! Das Projekt sammelte im Zuge der inhaltlichen, organisatorischen und technischen Planung bei der Einrichtung dieses Prototyps wichtige Erfahrungen, die ebenfalls dokumentiert sind und Nachahmern den Einstieg erleichtern können. Allgemein gelten dürfte, was eine vorab durchgeführte Befragung bei potenziellen Nutzern ergab: es bestehen sehr unterschiedliche Wünsche hinsichtlich der Auflösung und Detailtreve der abgebildeten Karten. Die Spanne reicht von einfachen Views, die kostenlos zur Verfügung stehen sollen, bis zur Ubersichtsdarstellung und zu qualitativ hochwertigen Reproduktionen auf CD-ROM. Dass sie eine genave Reproduzierbarkeit ermöglichen und zugleich flexibel sind, ist besonderer Vorteil der digitalen Medien. Zugleich wird der Bestand der wertvollen Originale auf diese Weise zusätzlich gesichert.

Viele Universitäten und Institute haben bereits heute ihre Kartensammlungen durch Internetportale geöffnet; bleibt zu wünschen, dass noch mehr diesem positiven Beispiel folgen, und dass möglichst eindeutige Oberflächen die Handhabung erleichtern. Auf diese Weise ließ und lässt sich mancher in den Archiven schlummernder
Schatz für Forschung, Lehre und Anwendung heben oder zumindest besser erschließen.

Zum Kern jeden kartographischen Schaffens, ob auf traditionelle Weise oder mit dem Einsatz digitaler Technik, gehört der Vorgang der Generalisierung. Seine Bedeutung im Prozess der Kartenherstellung wird von Außenstehenden meist erheblich unterschätzt, beschränkt er sich doch keineswegs allein auf die elementaren Vorgänge einer geometrischen Vereinfachung von Formen. Versteht man Karte, Globus und kartenverwandte Darstellungen unter moderner Sichtweise als Modell der Umwelt, stellen sich beim Generalisieren zugleich alle grundsätzlichen Fragen der Reliabilität und Validität. Die Vision, einmal den gesamten Karteninhalt mit dem Einsatz eines EDV-Programmes vollständig und automatisch generalisieren zu können, hat sich bislang nicht erfüllt, im Gegenteil: Wenn man sich vor Augen hält, wie stark elektronische Karten regelmäßig überladen werden und wie klein z.B. die zur Verfügung stehende Fläche auf dem Display eines Telefons ist, dann kann man durchaus gespannt sein, welche neuen Erkenntnisse, Auswege oder Chancen die Wissenschaft anzubieten hat Isiehe Aufsatz Mengl.

Für alle Leserinnen und Leser, die nicht nach Berchtesgaden kommen konnten, zur Dokumentation der verdienstvollen Tätigkeit des vorbereitenden Ortsausschusses und um neve Anregungen für künftige Kongresse zu geben, berichten in diesem Heft mit Joachim Neumann und Wolfram Pobanz zwei "erfahrene alte Hasen" vom Verlauf des 50. DKTs. Die Schriftleitung dankt beiden ausdrücklich für ihre Bereitschaft, diese in guter Tradition stehende Aufgabe übernommen zu haben.

Ihr 